\title{
Test of a prototype neutron spectrometer based on diamond detectors in a fast reactor
}

\author{
M. Osipenko, F. Pompili, M. Ripani, M. Pillon, G. Ricco, B. Caiffi, R. Cardarelli, G. Verona-Rinati, and S. Argiro
}

\begin{abstract}
A prototype of neutron spectrometer based on diamond detectors has been developed. This prototype consists of a ${ }^{6} \mathrm{Li}$ neutron converter sandwiched between two CVD diamond crystals. The radiation hardness of the diamond crystals makes it suitable for applications in low power research reactors, while a low sensitivity to gamma rays and low leakage current of the detector permit to reach good energy resolution. A fast coincidence between two crystals is used to reject background. The detector was read out using two different electronic chains connected to it by a few meters of cable. The first chain was based on conventional charge-sensitive amplifiers, the other used a custom fast charge amplifier developed for this purpose. The prototype has been tested at various neutron sources and showed its practicability. In particular, the detector was calibrated in a TRIGA thermal reactor (LENA laboratory, University of Pavia) with neutron fluxes of $10^{8} \mathrm{n} / \mathrm{cm}^{2} \mathbf{s}$ and at the $3 \mathrm{MeV}$ D-D monochromatic neutron source named FNG (ENEA, Rome) with neutron fluxes of $10^{6} \mathbf{n} / \mathrm{cm}^{2} \mathbf{s}$. The neutron spectrum measurement was performed at the TAPIRO fast research reactor (ENEA, Casaccia) with fluxes of $10^{9} \mathrm{n} / \mathrm{cm}^{2} \mathrm{~s}$. The obtained spectra were compared to Monte Carlo simulations, modeling detector response with MCNP and Geant4.
\end{abstract}

Index Terms-neutron spectrometer, fast reactor, diamond detector

\section{INTRODUCTION}

$\mathbf{T}$ HE diamond detectors were proposed for measurements of neutrons in nuclear facilities already in the ' 60 s. This was motivated by the outstanding properties of diamond, among others its high radiation hardness and low intrinsic noise at high temperatures. In fact, the Wigner displacement energy necessary to remove an atom from diamond crystal is $43 \mathrm{eV}$ compared to $13-20 \mathrm{eV}$ of Silicon. Many studies were undertaken to estimate diamond radiation hardness [1] and concluded that it is at least an order of magnitude higher than that of Si. The bandgap of diamond is $5.5 \mathrm{eV}$ allowing only negligible concentration of carriers at room temperature, mostly created by impurities and crystal defects. All these advantages of diamond crystal were recognized long ago. However, only with advent of the CVD technique practical applications of diamond detectors became feasible. In fact, CVD diamond growth provides unprecedented crystal quality,

Manuscript received April 6, 2015. This work was supported in part by the Istituto Nazionale di Fisica Nucleare INFN-E strategic program.

M. Osipenko, M. Ripani and G. Ricco are with the Istituto Nazionale di Fisica Nucleare, Genoa, 16146 Italy (e-mail: osipenko@ge.infn.it).

B. Caiffi, was with Università di Genova, Genoa, 16146 Italy.

F. Pompili, M. Pillon and M. Angelone are with the ENEA, Frascati, 00044 Italy.

G. Verona-Rinati and R. Cardarelli are with Università di Roma Tor Vergata, Rome, 00133 Italy.

S. Argiro is with Università di Torino and INFN, Turin, 10125 Italy. mandatory for detector applications. In particular, the relative concentration of most common diamond impurities like $\mathrm{B}$ and $\mathrm{N}$ was reduced below $(1 \div 5) \times 10^{-9}$ [2]. Also a high quality polishing techniques were developed providing $\mathrm{nm}$ scale surface uniformity [2].

Fast nuclear reactors, like ADS or critical with $\mathrm{Na}$ cooling, feature a non-trivial neutron spectrum. This allows for a lower production rate of radioactive waste and the burn-out of a fraction of the dangerous actinides. Both the reactor dynamics and burn-out of fuel and actinides depend on the neutron spectrum. Conventionally the neutron spectrum in a reactor is measured by activation foil analysis. Indeed, the activation of an isotope can be related to the convolution of the neutron flux with the isotope activation cross section. However, this complex, off-line, procedure introduces large systematic uncertainties. A simple on-line technique is necessary for characterization of reactor transients. For this purpose we developed a novel neutron spectrometer based on ${ }^{6} \mathrm{Li}$ converter sandwiched between two CVD diamond detectors. The energy of the incident neutron converts completely into the energy of charged particles through the ${ }^{6} \mathrm{Li}(\mathrm{n}, \alpha) t$ reaction. This allows for event-by-event neutron energy measurement with the advantages of a solid state detector. Moreover, the ${ }^{6} \operatorname{Li}(\mathrm{n}, \alpha) t$ reaction is highly exothermic with $Q=4.7 \mathrm{MeV}$, which permits to reduce background by imposing a high detection threshold. The coincidence between two crystals suppresses further noise and competing reactions.

In the present article we describe the experiment performed at TAPIRO, which allowed, for the first time, a direct reconstruction of the fast reactor neutron spectrum.

\section{Spectrometer Description}

The spectrometer is based on the diamond detectors developed in Refs. [3], [4] at the "Tor Vergata" University. These detectors are composed of three main layers: p-type diamond, intrinsic diamond and metal contact. Such a scheme forms a diode-like structure. Once polarized in reverse bias mode by applying negative voltage on the p-type (B-doped) cathode, a depletion layer is created in the intrinsic diamond. The degenerate p-type layer acts as an ohmic contact. Instead, the anode, creates a Schottky junction with the underlying intrinsic diamond. In this configuration the electric current generated by the passage of an ionizing particle in the depletion layer flows across the detector without any barrier. In fact, the Schottky junction at the intrinsic diamond-metal interface accelerates electrons leaving the diamond bulk.

The LiF compound was chosen as a neutron converter because of its chemical neutrality. The selected LiF was 


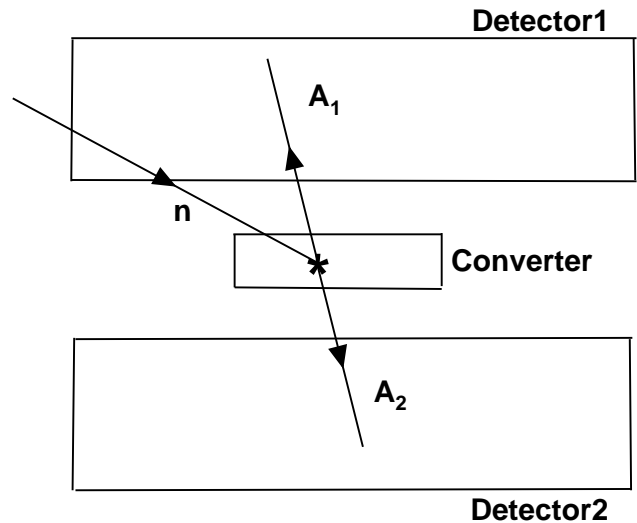

Fig. 1. Conceptual design of the spectrometer: the incident neutron is converted into two charged particles inside the converter layer and these products are measured by two diamond detectors.

enriched with ${ }^{6} \mathrm{Li}$ isotope to $96 \%$. The $\mathrm{LiF}$ layer is deposited on the top of metallic anode in such a way that $\alpha$ and $t$ can easily penetrate into the intrinsic diamond depletion layer with minimal loss of energy. Then the two identical detectors, called SCD282 and SCD240, were placed with their LiF layers one in front of the other leaving only $50 \mu \mathrm{m}$ gap for the central ground microwire as shown in Fig. 1 .

\section{TAPIRO NEUtRon FluX MEASUREMENTS}

In order to prove capability of the spectrometer of measuring neutron spectrum in a fast reactor the irradiation in TAPIRO was performed at ENEA of Casacia [5]. TAPIRO is a fast source reactor with maximum power of $5 \mathrm{~kW}$ which features an almost fission-like neutron spectrum with flux of $3 \times 10^{12} \mathrm{n} / \mathrm{cm}^{2} \mathrm{~s}$ in its core. The core made of $93.5 \%$ enriched ${ }^{235} \mathrm{U}(98.5 \%)$ - Mo (1.5\%) alloy is shaped as a cylinder with diameter of $12 \mathrm{~cm}$ and $12 \mathrm{~cm}$ tall. The reflector, divided in two parts, inner - up to $18 \mathrm{~cm}$ and outer - up to $40 \mathrm{~cm}$, is made of electrolytic copper. This is followed by a biological shield, $1.75 \mathrm{~m}$ thick borated concrete. For the experiments the reactor features 3 irradiation channels in the median plane of the core, radial channels 1 and 2 and diametral channel, one tangential channel crossing the entire reactor $5 \mathrm{~cm}$ above it core and the thermal column. The mean neutron energy decreases rapidly with the distance from the core or the depth in the reflector, allowing to select the desired spectrum by choosing the irradiation point.

\section{A. Experimental Setup}

The spectrometer was installed in the TAPIRO's tangential channel at the closest point to the core (fast position). At this location the tangential channel has a diameter of $3 \mathrm{~cm}$, which gives the depth into the reactor inner reflector of 2.8-5.8 $\mathrm{cm}$ and the radial distance from the core center of $10.6 \mathrm{~cm}$. This irradiation point is shown in Fig. 2] According to MCNP simulations [6], [7] shown in Fig. 3 the mean neutron energy in this location is $\left\langle E_{n}>\simeq 0.586 \mathrm{MeV}\right.$ and the total neutron flux $\phi_{n}^{\text {tot }} \simeq 1.89 \times 10^{9} \mathrm{n} / \mathrm{cm}^{2} / \mathrm{s} / 10 \mathrm{~W}$.

For a better determination of resolution, for energy calibrations and for comparison between two neutron spectra the

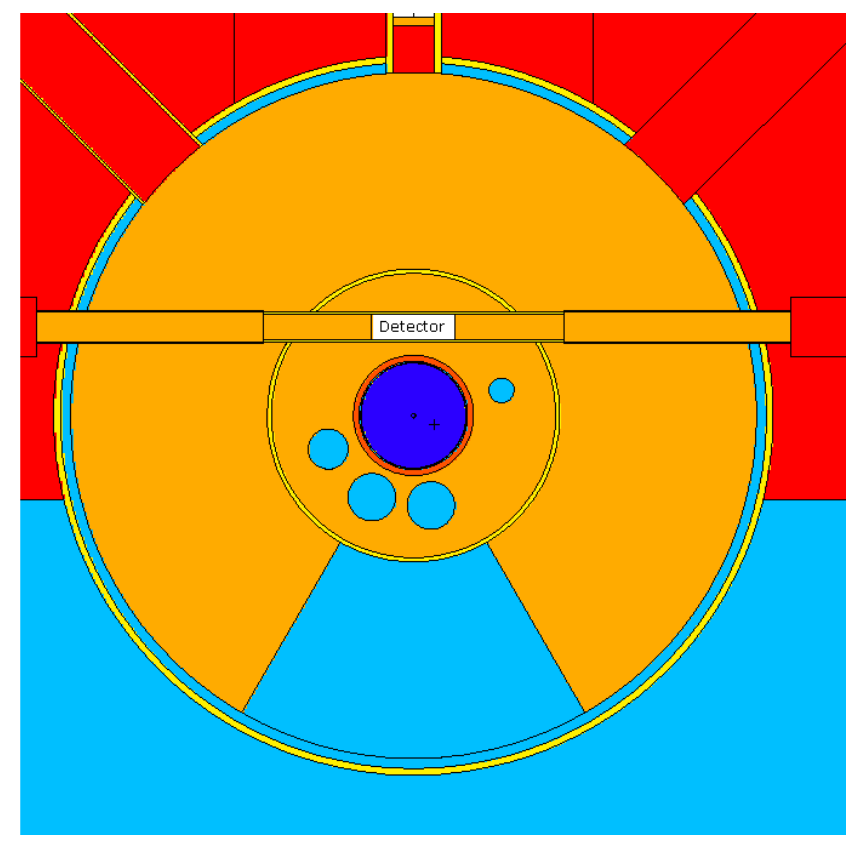

Fig. 2. Spectrometer location in the TAPIRO reactor (top view). The dark blue color indicates the reactor core, yellow circles - inner and outer reflector, long yellow rectangle - Tangential channel, the detector is represented by the short white rectangle near the core.

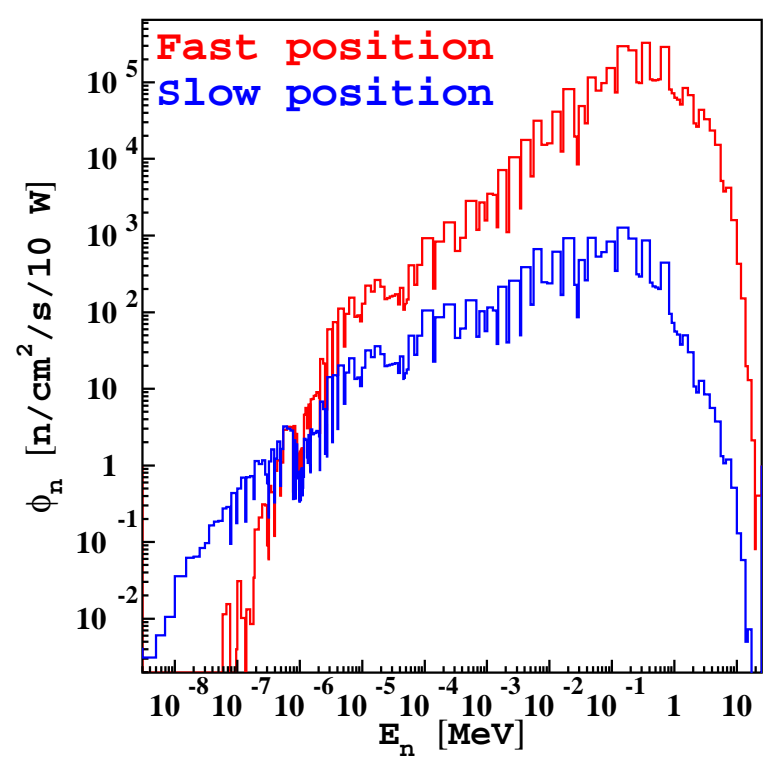

Fig. 3. Neutron fluxes in the two irradiation positions expected from MCNP simulations [6] at $10 \mathrm{~W}$ reactor power.

spectrometer was also irradiated in the second (slow) position. This second irradiation was performed with the tangential channel plug retracted by $30 \mathrm{~cm}$ almost to the external edge of the outer reflector. This position was called slow because of the significantly lower mean neutron energy $\left\langle E_{n}\right\rangle \simeq 0.166$ $\mathrm{MeV}$. The total flux in this location is expected to be $\phi_{n}^{\text {tot }} \simeq$ $1.28 \times 10^{8} \mathrm{n} / \mathrm{cm}^{2} / \mathrm{s} / 10 \mathrm{~W}$. The comparison of the fluxes in the two irradiation positions is shown in Fig. 3.

The two sandwich spectrometer outputs were connected via 
a $5 \mathrm{~m}$ RG62 cable to custom fast charge amplifiers [8]. At the amplifier inputs fast DC decouplers were used to connect the detector bias voltage of $-80 \mathrm{~V} /-120 \mathrm{~V}$, corresponding to $1.5-2$ $\mathrm{V} / \mu \mathrm{m}$, supplied by an Ortec 710 module [9]. The detector's signals boosted and shaped in the primary amplifiers were further amplified by a Philips Scientific 771 amplifier [10] with voltage gain set to 4 . The overall gain of this chain was about $100 \mathrm{pVs} / \mathrm{fC}$. The amplified signals were sent to a SIS3305 10-bit digitizer [11] working in $5 \mathrm{Gs} / \mathrm{s}$ mode. The digitizer internal trigger was configured to fire on a coincidence [12] between the two spectrometer channels within a $64 \mathrm{~ns}$ interval. The individual channel threshold was set to 40 $\mathrm{mV}$. The Data AcQuisition (DAQ) system was based on VME modular electronics. In particular, a Concurrent Tech. VX81309x single board computer [13] was used as VME controller as well as the acquisition host. The VX813-09x run 32-bit Centos 6 Linux operating system with native Tsi148 VME controller drivers. The SIS3305 was configured to generate interrupts on the VME bus when the number of events in its $2 \mathrm{~Gb}$ buffer exceeded the imposed threshold. When the interrupt was received by the VX813/09x controller all the data were copied from SIS3305 buffer through a fast DMA MBLT transfer and sent to a secondary DAQ thread. This secondary DAQ thread was buffering and saving the data on a fast Compact Flash card. For every event $N_{\text {samples }}=960$ samples were saved in each channel for the total waveform duration of $192 \mathrm{~ns}$. Among these samples the signal length was $L_{\text {signal }} \sim 130$ samples or $25 \mathrm{~ns}$. Two TDC values of the common trigger as seen in the two channels were also saved. The amount of data (about $240 \mathrm{~kb} /$ event) and event rate were relatively small, thus VME transfer and disk writing speed were sufficient to run the acquisition without additional delays.

During the experiment the reactor power was continuously monitored by a fission chamber. Although the reactor power was fairly stable during irradiation the fission chamber current was saved to the experiment log file for better accuracy.

\section{B. Data Analysis}

For the absolute normalization of the measurement the TAPIRO power monitoring system combined with the powerto-flux conversion from Refs. [14], [6] were used. The correlation between sandwich spectrometer event rate and fission chamber current, shown in Fig. 4, was found to be linear within uncertainties on the interval of one order of magnitude. This normalization allows to correlate the measured absolute neutron flux to a given reactor power and to compare this to the previous measurements [14].

Although the coincidence trigger was fairly efficient in suppressing noise the saved data were further filtered to discard remaining backgrounds. This procedure included the following checks: the event length (in samples) was checked to be the nominal one, the integral of the first 100 samples before the signal were checked to be equal to the baseline within $3 \sigma$, the trigger sample was checked to lay at the sample number $>50$ and $<N_{\text {samples }}-L_{\text {signal }}-50$, the time of arrival of the signal from the two detectors was requested to lay within $4.8 \mathrm{~ns}$ (1 ADC clock), the integral of the signal peak had to

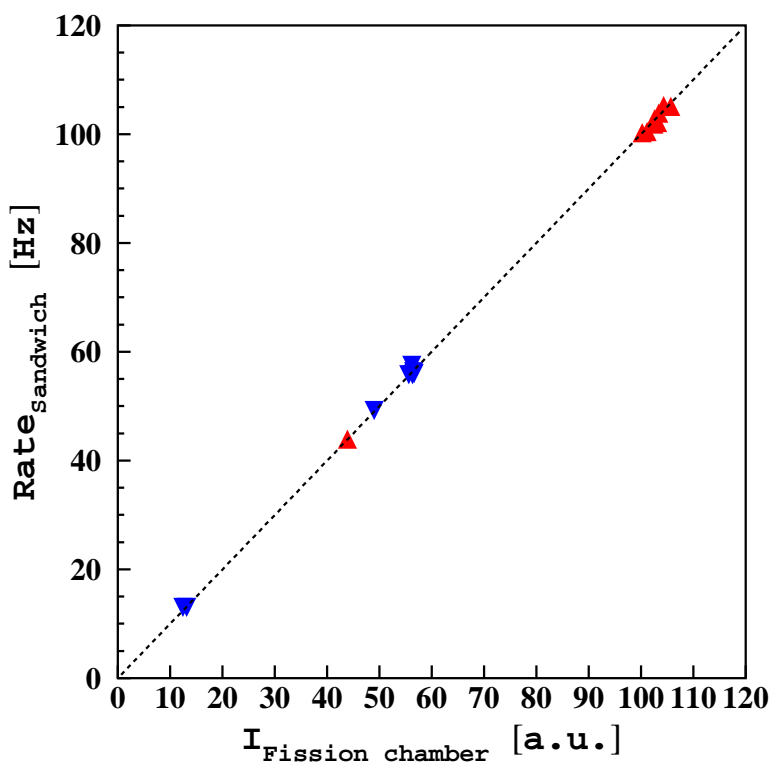

Fig. 4. Correlation between the spectrometer event rate and the current of the power monitoring fission chamber. Measurements in different positions are indicated by different colors.

be above the threshold in both channels. All these conditions discarded $<1 \%$ of the recorded events.

The calibration of the energy deposited in each crystal was based on the energy of the produced $t$, corrected for the energy lost in LiF layer and $\mathrm{Cr}$ anode. The digitizer baseline was taken as the zero energy point. The width of the $t$-peak was found to be $87 \mathrm{keV}$ for SCD282 and $104 \mathrm{keV}$ for SCD240. These values were only slightly larger than the measured electronic's noise RMSs of 70 and $77 \mathrm{keV}$, respectively, while the intrinsic broadening of the resolution due to the energy loss in LiF layer and $\mathrm{Cr}$ contacts was estimated to be about $5 \mathrm{keV}$.

The sum of the two energies deposited in the two crystals gives the incident neutron energy plus $Q$-value of the reaction. The energy of thermal neutrons is smaller than the energy resolution of the detector and therefore in this case the total measured energy is close to the reaction $Q$-value. The spectrum of the sum of the energies of the two crystals builds as an asymmetric peak at around $4.7 \mathrm{MeV}$. The peak width was found to be $160 \mathrm{keV}$, compared to the intrinsic resolution of $21 \mathrm{keV}$ due to energy losses in LiF layer and $\mathrm{Cr}$ contacts.

During the experiment it was noticed that the energy calibrations of two diamond detectors changed with time as shown in Fig 5. This effect was attributed to the build-up of space charge at one of the two electrical contacts of intrinsic diamond: p-type (B-doped) layer or $\mathrm{Cr}$ anode. The build-up of space charge near one contact screens the contact's potential, thus reducing the electric field across the diamond depletion layer. This, in turn, leads to a modification of the electrical signal shape, damping its height and stretching its length. Although the total collected charge remains the same a fraction of it could be cut off by the finite integration window. Choosing a longer signal integration time allows to compensate this effect, but with the amplifier used in this experiment it leads to an unacceptable degradation of energy resolution. Therefore, 


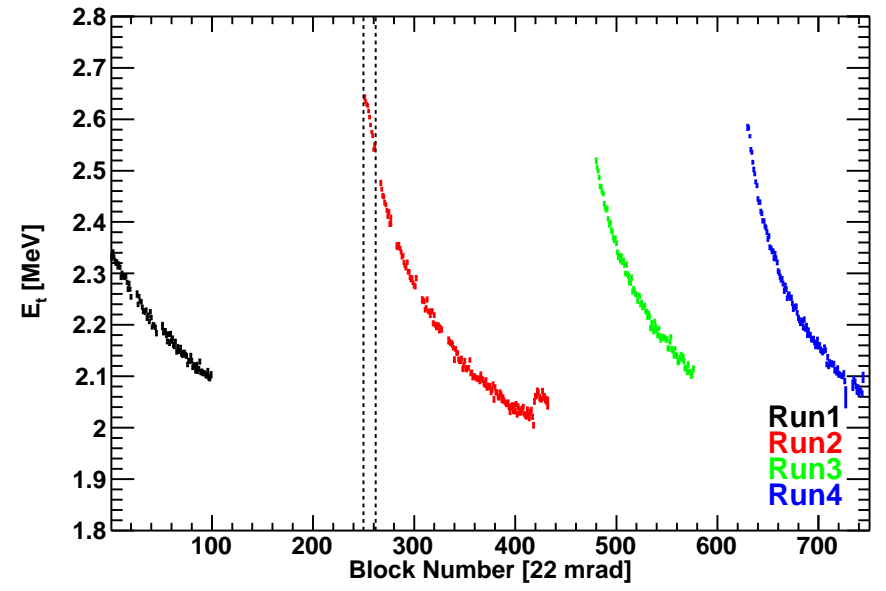

Fig. 5. Drift of the $t$ peak position with absorbed $\alpha+t$ dose. Detector bias supply was turned off between the runs shown by different colors. Only the fraction of data selected by the two dashed lines was kept in the present analysis.

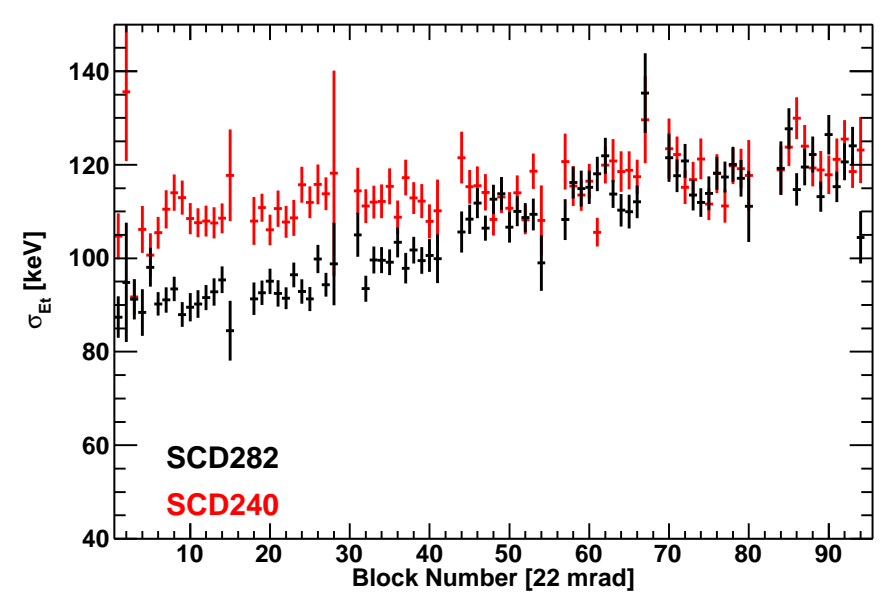

Fig. 6. Drift of the $t$ peak resolution with absorbed $\alpha+t$ dose for the two diamond detectors of the spectrometer.

to correct for this drift of charge collection within fixed integration gate, time-dependent calibration constants were applied. The drift affected not only the energy calibration, but also the spectrometer resolution. Indeed, a constant resolution in terms of signal charge after application of time-dependent energy calibrations becomes time-dependent as well, as shown in Fig. 6. In order to maintain the best energy resolution only a small fraction, about $2 \%$, of the data indicated by the two dashed lines in Fig. 5 was selected for this analysis.

It is worth noting that the drift of the observed charge collection is recoverable by turning off the bias voltage. Indeed, in Fig. 5 the four different colors represent the distributions taken in different runs, in between of which the bias voltage was off. This observation confirms our interpretation of the drift as the space charge effect. Another confirmation comes

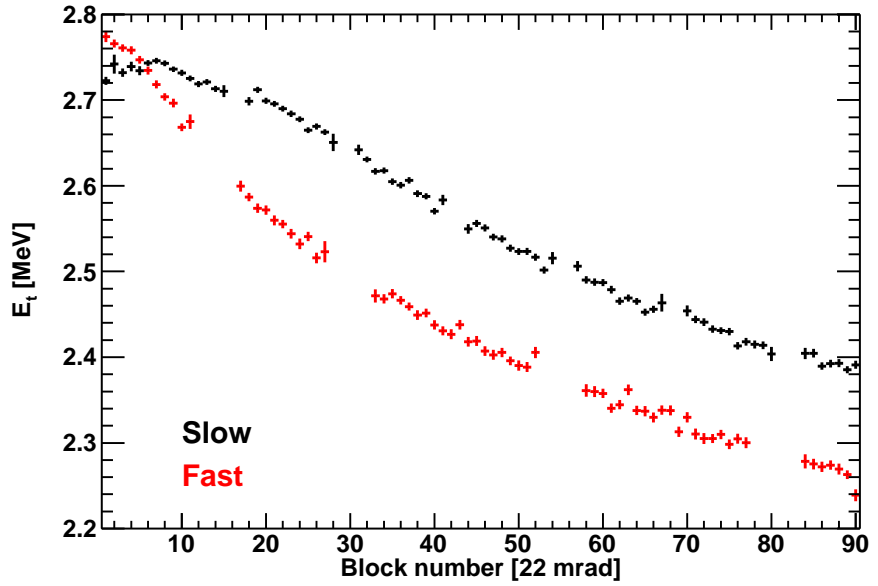

Fig. 7. Comparison of the drifts of the $t$ peak position in fast and slow neutron spectra. The elastic scattering contribution is not included in the dose on $X$-axis.

from the comparison of the drifts in slow and fast neutron fluxes shown in Fig. 7. The produced space charge depends on the dose deposited in the diamond depletion layer. At the same dose produced by $\alpha$ and $t$ particles given in $X$-axis the fast neutron spectrum generated a larger dose due to neutron elastic scattering off the carbon nuclei. Thus in the fast neutron flux a more rapid drift was observed.

The absolute amount of ${ }^{6} \mathrm{Li}$ deposited by evaporation on the diamond's $\mathrm{Cr}$ contacts was determined in a dedicated experiment [15] with $20 \%$ accuracy. This value was used in the simulations to determine the correct absolute efficiency of the spectrometer.

The sandwich detector response to the neutron flux was modeled by the Geant Monte Carlo library [16] version 4.9.5.p01. The detector was described in details including: PCB support, HPHT diamond substrate, B-doped CVD diamond cathode substrate, intrinsic CVD diamond, $\mathrm{Cr}$ anode layer, $\mathrm{LiF}$ layer, $\mathrm{Au}$ deposited layers and $\mathrm{Au}$ coated copper wires.

The neutron flux of the TAPIRO fast reactor in the two irradiation positions taken from Ref. [6] was generated isotropically on the spherical surface around the spectrometer of area about $1 \mathrm{~cm}^{2}$. The events with the energy deposited in both crystals of the sandwich sensitive volume above the threshold were selected.

In order to normalize the simulations to the same neutron flux, seen by the spectrometer, the reconstructed Geant 4 distributions were rescaled by the factor:

$$
L_{\text {sim }}=\phi_{n} t_{\text {run }} \frac{S_{g e n}}{N_{g e n}},
$$

where $\phi_{n}$ is the neutron flux monitored by TAPIRO fission chamber, $t_{r u n}$ is DAQ runtime, $N_{\text {gen }}$ is the total number of neutrons generated on the surface of area $S_{g e n}$.

In order to compare Geant 4 simulations to the data an additional Gaussian smearing due to electronic readout noise was 


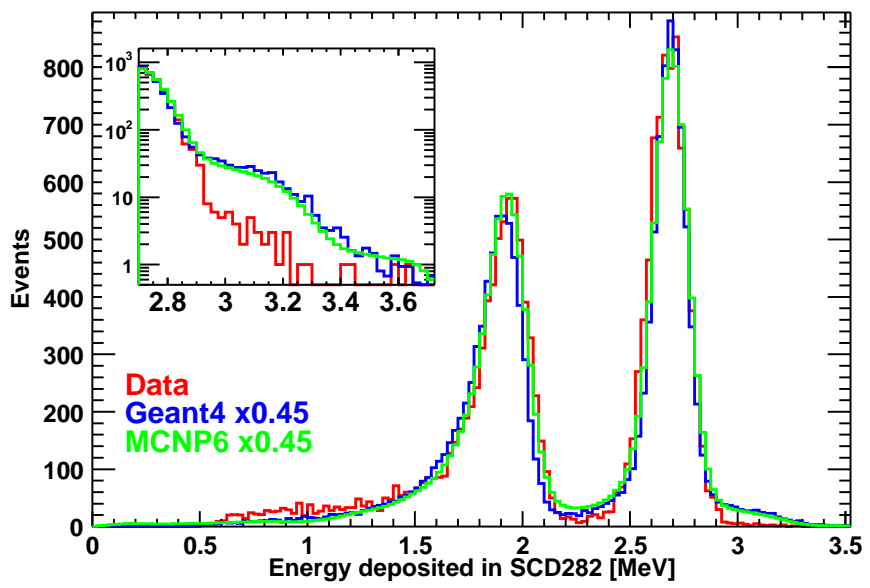

Fig. 8. Energy deposited in SCD282 of the spectrometer in comparison to Geant 4 and MCNP6 simulations for the slow position. Because of the discrepancy in the absolute values between expected and measured fluxes both simulations were rescaled by the factor 0.45 .

added to the reconstructed energies. The obtained simulations describe the data fairly well, in particular in the region of $t$ peak, which has almost a Gaussian shape. Instead the $\alpha$-peak is more asymmetric and features a larger 1.h.s. tail due to a higher energy loss of $\alpha$ particles. The total deposited energy distribution is also well reproduced.

Similar simulations were performed with MCNP6 [17], but with $\mathrm{Cr}$ contacts of $100 \mathrm{~nm}$ thickness which determined a larger energy loss of $\alpha$ and slight shift in its peak position.

\section{Results}

The data measured in the slow neutron flux position were analyzed first in order to calibrate better the spectrometer response in terms of deposited energy. The energy deposited in a single spectrometer crystal was compared to Geant 4 and MCNP6 simulations as shown in Fig. 8 Both simulations were obtained by generating neutron flux expected for this location from Ref. [6] and normalized in absolute value to the integrated flux of TAPIRO fission chamber. However, this comparison revealed that the measured flux was a factor of two lower than expected. Thus all the simulations were renormalized by the factor 0.45 for better qualitative comparison with the data. Moreover, in the zoomed part of the spectrum around 2.9-3.3 MeV a significant difference between data and rescaled simulations was found suggesting further suppression of the measured flux in the neutron energy region $0.2-0.5 \mathrm{MeV}$ by a factor 4 .

The total energy deposited in the two crystals of the spectrometer, shown in Fig. 9 , exhibits similar behavior. Also here both Geant 4 and MCNP simulations were rescaled by the factor 0.45 for a better comparison. The high energy tail generated by neutrons with energies of $0.2-0.5 \mathrm{MeV}$, corresponding here to 4.9-5.2 MeV deposited energy, is also strongly overestimated by the simulated flux. Another difference, not so evident in the single crystal spectrum, is present in

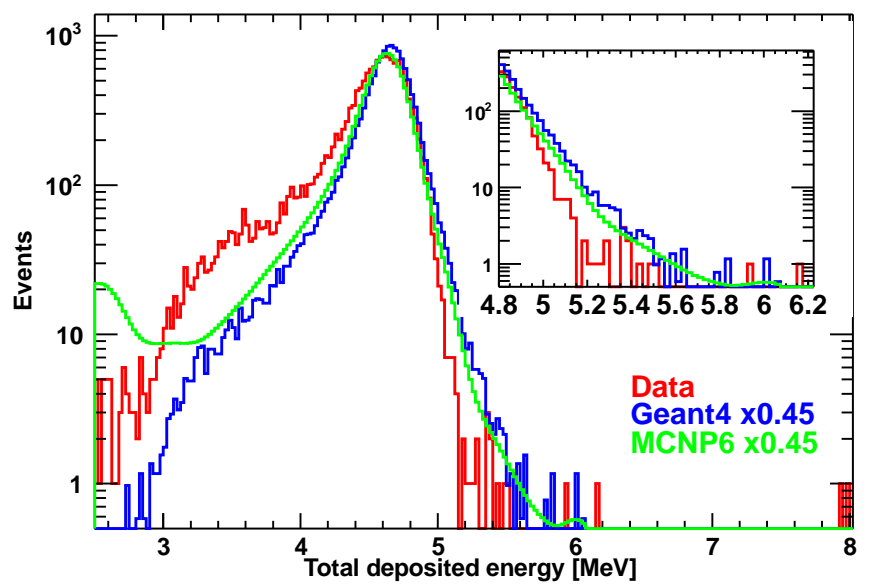

Fig. 9. Total energy deposited in the spectrometer in comparison to Geant4 and MCNP6 simulations for the slow position. Because of the discrepancy in the absolute values between expected and measured fluxes both simulations were rescaled by the factor 0.45 .

the low energy tail. The ideal uniformity of $\mathrm{Cr}$ anode assumed in the simulations does not describe its real distribution. Although the average $\mathrm{Cr}$ anode thickness is well reproduced, as one can see from the comparison of the positions of the $\alpha$ peak in data and simulations, small regions of thicker anode determine a larger $\alpha$ energy loss. $t$ is less sensitive to this disuniformity because of its lower energy loss rate in matter.

It is possible to extract the neutron flux using the distribution of the total deposited energy. To this end the data and simulation distributions were rebinned in larger energy bins, accounting for the spectrometer resolution. The achieved resolution of $160 \mathrm{keV}$ on the total deposited energy allowed to use $400 \mathrm{keV}$ bins, which corresponded roughly to the FWHM. The measured flux $\phi_{n}^{e x p}$ in each energy bin was obtained as the ratio:

$$
\frac{\partial \phi_{n}^{e x p}}{\partial E_{n}}\left(E_{n}\right)=\frac{\partial \phi_{n}^{\text {sim }}}{\partial E_{n}}\left(E_{n}\right) \frac{N_{\text {data }}\left(E_{n}\right)}{L_{\text {sim }} N_{\text {sim }}\left(E_{n}\right)},
$$

where $\phi_{n}^{\text {sim }}$ is the simulated neutron flux, $N_{\text {data }}\left(E_{n}\right)$ and $N_{\text {sim }}\left(E_{n}\right)$ are the numbers of data and simulation events in the given energy bin. The obtained flux is shown in Fig. 10 in comparison with MCNP simulations from Ref. [6] (blue) and older evaluations from Ref. [18] (green) and Ref. [19] (black). The data from Ref. [19] were rescaled due to position mismatch by the factor 0.17 , obtained as the ratio of MCNP simulations in the two points.

The same analysis was performed on the data taken in the fast neutron flux position. These results are shown in Figs. 11, 12 and 13 Qualitatively the fast position measurement showed larger disagreement with the flux from Ref. [6]. Although the integrated neutron flux, which comes mostly from the first energy bin was compatible within systematic uncertainties. In fact, already the distribution of the energy deposited in a single crystal, shown in Fig. 11, demonstrated a wrong ratio of fast and thermal neutrons. In the total deposited energy distribution 


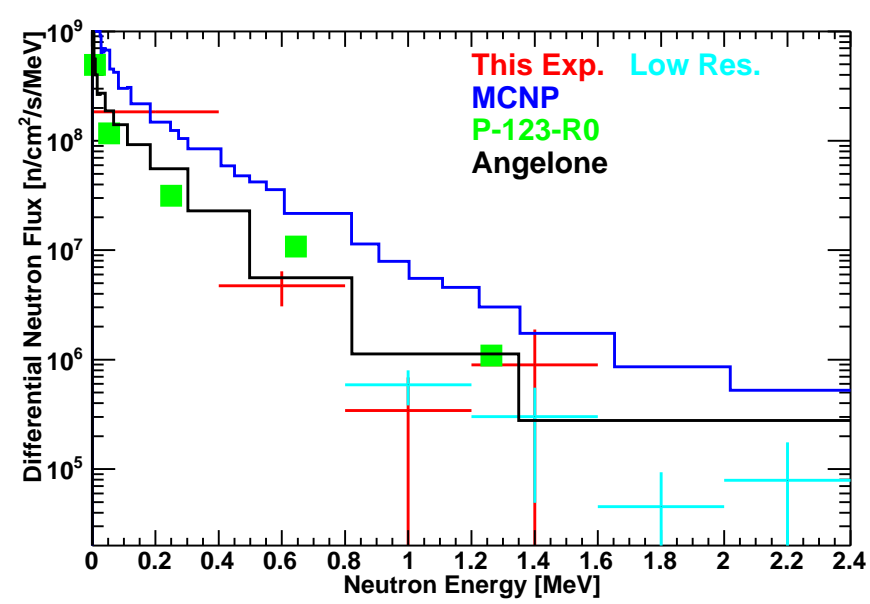

Fig. 10. Measured neutron flux in the slow position at $12 \mathrm{~kW}$ reactor power in comparison with MCNP simulations from Ref. [6] (blue) and older evaluations from Ref. [18] (green) and Ref. [19] (black). The latter distribution was rescaled by the factor 0.17 due to position mismatch. The cyan data points are from a low resolution but high statistics run. The data error bars are statistical only, the systematic uncertainty consists of and overall $20 \%$ error due to ${ }^{6} \mathrm{Li}$ quantity plus an energy dependent part due to spectrometer resolution description.

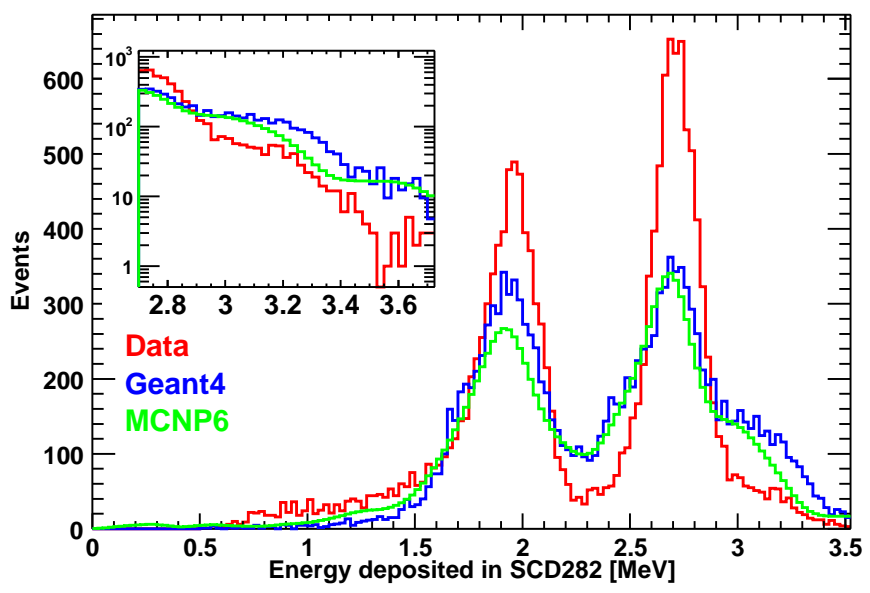

Fig. 11. Energy deposited in SCD282 of the spectrometer in comparison to Geant4 and MCNP6 simulations for fast position.

from Fig. 12 the difference resulted in an overall shift of the simulated distribution with respect to the measured one.

The reconstructed neutron flux, shown by red points in Fig. 13, is a factor of 5 lower at neutron energies above 0.4 $\mathrm{MeV}$. This measured flux was used to repeat the unfolding procedure [14] by means of SAND II [20] code. The unfolding resulted in the flux shown in Fig. 13 by magenta histogram, which is in good agreement with the flux measured by our spectrometer up to $2 \mathrm{MeV}$ (red points). Above $2 \mathrm{MeV}$ the data points from a low resolution but high statistics run were added. These additional points disagree with activation analysis.

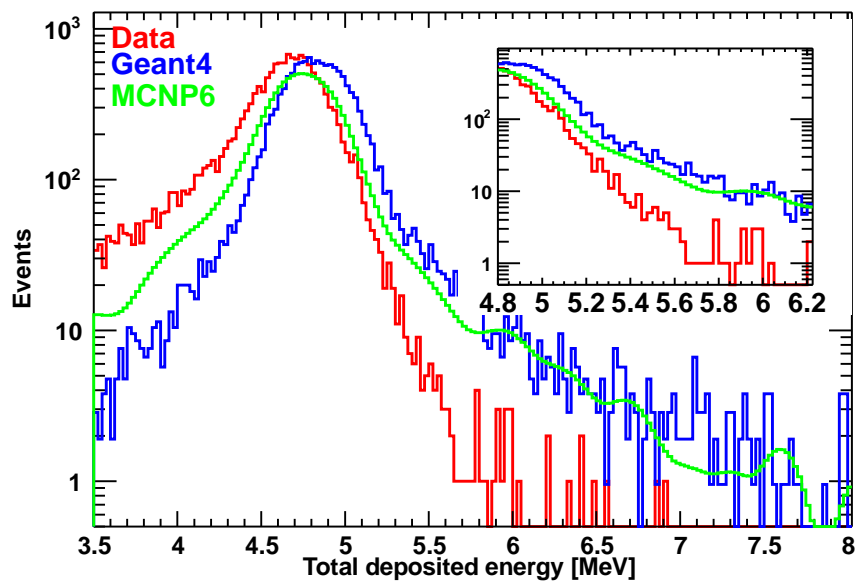

Fig. 12. Total energy deposited in the spectrometer in comparison to Geant4 and MCNP6 simulations for fast position.

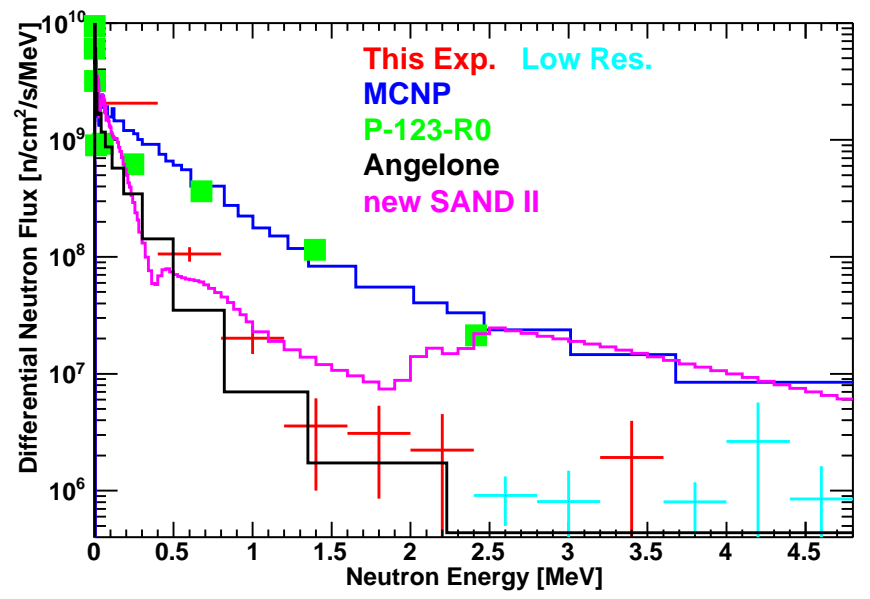

Fig. 13. Measured neutron flux in the fast position at $5 \mathrm{~kW}$ reactor power in comparison with MCNP simulations from Ref. [6] normalized to activation foil analysis [14] (blue), repeated SAND II activation analysis with modified input flux (magenta) and older evaluations from Ref. [18] (green) and Ref. [19] (black). The latter distribution was rescaled by the factor 2.56 due to position mismatch. The cyan data points are from a low resolution but high statistics run. The data error bars are statistical only, the systematic uncertainty consists of and overall $20 \%$ error due to ${ }^{6} \mathrm{Li}$ quantity plus an energy dependent part due to spectrometer resolution description.

\section{CONClusions}

A novel neutron spectrometer for fast nuclear reactors based on ${ }^{6} \mathrm{Li}$ converter sandwiched between two CVD diamond detectors was used to measure neutron spectrum in the TAPIRO fast reactor. The spectrometer was installed in the TAPIRO's tangential channel in two different positions: fast position $5 \mathrm{~cm}$ in the reflector and slow position - at the edge of the reflector. According to MCNP simulations [6] in these positions the neutron fluxes of $\phi_{n}^{\text {tot }} \simeq 1.89 \times 10^{9} \mathrm{n} / \mathrm{cm}^{2} / \mathrm{s} / 10$ $\mathrm{W}$ and $\phi_{n}^{\text {tot }} \simeq 1.28 \times 10^{8} \mathrm{n} / \mathrm{cm}^{2} / \mathrm{s} / 10 \mathrm{~W}$ with mean neutron 
energies $<E_{n}>\simeq 0.586 \mathrm{MeV}$ and $\left\langle E_{n}>\simeq 0.166 \mathrm{MeV}\right.$ were expected. The measured fluxes were found to be different by a factor 0.45 at the slow position and by a factor 0.9 at the fast position with $20 \%$ overall systematic uncertainty. Moreover, the neutron spectra in both positions resulted to be considerably softer, such that $\left\langle E_{n}\right\rangle^{e x p} \sim 0.25 \mathrm{MeV}$ (fast) and $\left\langle E_{n}>^{\exp } \sim 0.1 \mathrm{MeV}\right.$ (slow). The absolute normalization of MCNP simulations from Ref. [6] was obtained from the activation foil irradiation at $3.5 \mathrm{~kW}$ reactor power in the radial channel 1 at $5 \mathrm{~cm}$ in the reflector [14]. Therefore, the expected fluxes come from an extrapolation of the measured spectrum into different locations and to different reactor power. Although the distance from the reactor core in Ref. [14] is similar to the current one (fast position) the radial channel 1 is located at the middle of the core, while the tangential channel passes above it. This leads to a harder spectrum in Ref. [14]. This difference can be not completely reproduced by MCNP model. Moreover, high resolution data were sensitive only to the neutron energy range $100 \mathrm{keV}<E_{n}<2 \mathrm{MeV}$, while the activation foil analysis [14] did not have enough sensitivity in this interval. Indeed, the $5 \%$ sensitivity ranges given by SAND II unfolding code for the irradiated isotopes excluded completely the region $230 \mathrm{keV}<E_{n}<2.5 \mathrm{MeV}$. In order to verify this statement the unfolding analysis was repeated with a new input flux modified according to the values measured in this article. The analysis converged to the solution compatible with our high resolution data. The data measured with low resolution extend the covered energy range up to $4.8 \mathrm{MeV}$. In this extended region $2.5 \mathrm{MeV}<E_{n}<4.8 \mathrm{MeV}$ our data are incompatible with Ref. [14]. This could be explained also by the difference in reactor power, 700 times lower in the present experiment.

It must be emphasized that because of rapid fall of cross section with energy the measured absolute flux in the first bin is highly dependent on the initial neutron spectrum below $1 \mathrm{keV}$. Because of the observed space charge build-up the analysis was performed only on $2 \%$ of the measured data featuring the best energy resolution. With such statistics the neutron spectrum reconstruction was limited to the region $E_{n}<2 \mathrm{MeV}$. The spectrometer energy resolution of $160 \mathrm{keV}$ allowed for neutron spectrum reconstruction in $400 \mathrm{keV}$ bins. The resolution of a single crystal was two times better and, in fact, it constrained much better the high energy part of the spectrum by the comparison to simulations. However, the single crystal spectrum does not allow to reconstruct neutron energy on a event-by-event basis.

The experiment had shown the following limitations of the present spectrometer prototype:

- the space charge build-up does not permit for a long continuous acquisition and therefore limits the statistics,

- relatively low energy resolution prevents from using narrow energy bins.

All these limitations will be addressed in the future prototypes.

\section{ACKNOWLEDGMENT}

Authors would like to acknowledge excellent support provided during the experiment by the TAPIRO facility staff and technical services of ENEA (Casaccia). We also want to acknowledge the useful discussions with M. Carta and help with neutron flux simulations from A. Santagata (ENEA, Casaccia).

\section{REFERENCES}

[1] W. de Boer et al., Phys. Status Solidi, vol. 204, p. 3009, 2007.

[2] "Element six, electronic grade cvd diamonds." [Online]. Available: http://www.e6cvd.com/cvd/page.jsp?pageid=415

[3] M. Angelone et al., "Neutron detectors based upon artificial single crystal diamond." IEEE TRANSACTIONS ON NUCLEAR SCIENCE, 2009, vol. 56, p. 2275.

[4] S. Almaviva et al., "Thermal neutron dosimeter by synthetic single crystal diamond devices." Applied Radiation and Isotopes, 2009, vol. 67, p. 183

[5] "ENEA, centro ricerche casaccia." [Online]. Available: http://www.enea.it/it/centro-ricerche-casaccia/

[6] N. Burgio, L. Cretara, M. Frullini, A. Gandini, V. Peluso, and A. Santagata, "Monte carlo simulation analysis of integral data measured in the SCK-CEN/ENEA experimental campaign on the TAPIRO fast reactor. experimental and calculated data comparison." Nuclear Engineering and Design, 2014, vol. 273, p. 350.

[7] N. Burgio, A. Santagata, and M. Frullini, "Validation of the monte carlo model of TAPIRO fast reactor using MCNPX on ENEA-GRID/CRESCO facility." ENEA, High Performance Computing on CRESCO infrastructure: research activities and results 2013, 2015.

[8] R. Cardarelli, A. D. Ciaccio, and L. Paolozzi, Nucl. Instr. and Meth. A, vol. 745, p. 82, 2014.

[9] "ORTEC." [Online]. http://www.ortec-online.com/download/710.pdf

[10] "Phillips [Online]. Avientific." Available: http://www.phillipsscientific.com/preview/770pre.htm

[11] "Struck innovative systeme gmbh." [Online]. Available: http://www.struck.de/sis3305.html

[12] M. Osipenko et al., "INFN preprint, INFN-13-17-GE," 2014.

[13] "Concurrent technologies." [Online]. Available: http://www.gocct.com/sheets/VX/vx81x09x.htm

[14] M. Pillon, "ENEA report, RT-IVVS2-08," 2014.

[15] M. Osipenko et al., Nucl. Instr. and Meth. A, 2015.

[16] S. Agostinelli et al., Nucl. Instr. and Meth., vol. 506, p. 250, 2003.

[17] R. Forster et al., Nucl. Instr. and Meth., vol. 213, p. 82, 2004.

[18] "CNEN report, DIFIR(64), reattore sorgente veloce TAPIRO: programma sperimentale," 1964.

[19] M. Angelone et al., "CMS NOTE 1998/060," 1998.

[20] W. McElroy, S. Berg, T. Crockett, and R. Hawkins, "A computerautomated iterative method for neutron flux spectra determination," 1967, report AFWL-TR 67-41. 